\title{
Comparison of clinical outcomes after drug-eluting balloon and drug-eluting stent use for in-stent restenosis related acute myocardial infarction: A retrospective study
}

\author{
Chih-Yuan Fang ${ }^{1}$, Hsiu-Yu Fang ${ }^{1}$, Chien-Jen Chen ${ }^{1}$, Cheng-Hsu Yang ${ }^{1}$, Chiung-Jen Wu ${ }^{1}$, Wei-Chieh Lee \\ Corresp. 1 \\ ${ }^{1}$ Division of Cardiology, Department of Internal Medicine, Kaohsiung Chang Gung Memorial Hospital, Chang Gung University College of Medicine, \\ Kaohsiung, Taiwan \\ Corresponding Author: Wei-Chieh Lee \\ Email address: leeweichieh@yahoo.com.tw
}

Background

Good results of drug-eluting balloon (DEB) use are achieved in in-stent restenosis (ISR) lesions, small vessel disease, long lesions, and bifurcations. However, few reports exist about DEB use in acute myocardial infarction (AMI) with ISR. This study's aim was to evaluate the efficacy of DEB for AMI with ISR.

Methods

Between November 2011 and December 2015, 117 consecutive patients experienced AMI including STsegment elevation $\mathrm{MI}$, and non-ST-segment elevation MI due to ISR, and received percutaneous coronary intervention ( $\mathrm{PCl}$ ). We divided our patients into two groups: (1) PCl with further DEB, and (2) PCl with further drug-eluting stent (DES). Clinical outcomes such as target lesion revascularization, target vessel revascularization, recurrent $\mathrm{MI}$, stroke, cardiovascular mortality, and all-cause mortality were analyzed.

Results

Patients' average age was $68.37 \pm 11.41$ years; $69.2 \%$ were male. A total of 75 patients were enrolled in the DEB group, and 42 patients were enrolled in the DES group. The baseline characteristics between the two groups were the same without statistical differences except gender. Peak level of cardiac biomarker, pre- and post-PCl cardiac function were similar between two groups. The major adverse cardiac cerebral events rate (34.0\% vs. $35.7 \% ; p=0.688)$ and cardiovascular mortality rate $(11.7 \%$ vs. $12.8 \% ; p=1.000)$ were similar in both groups.

Conclusions

DEB is a reasonable strategy for AMI with ISR. Compared with DES, DEB an alternative strategy which yielded acceptable short-term outcomes and similar 1-year clinical outcomes. 
1 Comparison of clinical outcomes after drug-eluting balloon and drug-eluting stent use for

2 in-stent restenosis related acute myocardial infarction: a retrospective study

3

4 Chih-Yuan Fang, MD ${ }^{1 *}$, Hsiu-Yu Fang, MD ${ }^{1}$, Chien-Jen Chen, MD ${ }^{1}$, Cheng-Hsu Yang, MD ${ }^{1}$,

5 Chiung-Jen $\mathrm{Wu}, \mathrm{MD}^{1}$, Wei-Chieh Lee, $\mathrm{MD}^{1 *}$

6

$7{ }^{1}$ Division of Cardiology, Department of Internal Medicine, Kaohsiung Chang Gung Memorial

8 Hospital, Chang Gung University College of Medicine, Kaohsiung, Taiwan, Republic of China

$10 *$ Indicates equal contribution as first author.

11

12 Corresponding author: Wei-Chieh Lee, MD

Address for Correspondence: Division of Cardiology, Department of Internal Medicine,

Kaohsiung Chang Gung Memorial Hospital, 123, Ta Pei Road, Niao Sung District, Kaohsiung

City, 83301, Taiwan, Republic of China

Address for Contact: No.123, Ta Pei Rd, Niao-Sung district, Kaohsiung City, Taiwan

Tel: $+886-7-7317123$ ext. 2363

Fax: $+886-7-7322402$

E-mail: leeweichieh@yahoo.com.tw

22

23 Running title: Clinical outcomes after DEB Use for ISR related AMI 
25

26

27

28

29

30

31

32

33

34

35

36

37

38

39

40

41

42

43

44

45

\section{Abstract}

47 Background

48 Good results of drug-eluting balloon (DEB) use are achieved in in-stent restenosis (ISR) lesions, 
49 small vessel disease, long lesions, and bifurcations. However, few reports exist about DEB use in 50 acute myocardial infarction (AMI) with ISR. This study's aim was to evaluate the efficacy of 51 DEB for AMI with ISR.

52

53 Methods

54 Between November 2011 and December 2015, 117 consecutive patients experienced AMI 55 including ST-segment elevation MI, and non-ST-segment elevation MI due to ISR, and received 56 percutaneous coronary intervention (PCI). We divided our patients into two groups: (1) PCI with 57 further DEB, and (2) PCI with further drug-eluting stent (DES). Clinical outcomes such as target 58 lesion revascularization, target vessel revascularization, recurrent MI, stroke, cardiovascular 59 mortality, and all-cause mortality were analyzed.

61 Results

62 Patients' average age was $68.37 \pm 11.41$ years; $69.2 \%$ were male. A total of 75 patients were 63 enrolled in the DEB group, and 42 patients were enrolled in the DES group. The baseline 64 characteristics between the two groups were the same without statistical differences except 65 gender. Peak level of cardiac biomarker, pre- and post-PCI cardiac function were similar 66 between two groups. The major adverse cardiac cerebral events rate $(34.0 \%$ vs. $35.7 \%$; $=0.688)$ 67 and cardiovascular mortality rate $(11.7 \%$ vs. $12.8 \% ; p=1.000)$ were similar in both groups.

68

69 Conclusions

70 DEB is a reasonable strategy for AMI with ISR. Compared with DES, DEB an alternative 71 strategy which yielded acceptable short-term outcomes and similar 1-year clinical outcomes. 72 
75

76

77

78

79

80

81

82

83

84

85

86

87

88

89

90

91

92

93

94

95 Introduction

96 With the improvement of the technology and design of stent, the incidence of in-stent restenosis 
97 (ISR) decreased. Drug-eluting balloons (DEBs) have become an important alternative to the 98 current treatment of ISR (Wöhrle et al., 2013). The 2014 European guideline for coronary 99 revascularization recommends the use of DEB for the treatment of ISR of a bare-metal stent 100 (BMS) or drug-eluting stent (DES) (Class I, level of evidence: B) (Windecker et al., 2014).

101 However, the American College of Cardiology/American Heart Association/Society for 102 Cardiovascular Intervention guidelines for percutaneous coronary intervention (PCI) 103 recommends DES to treat BMS ISR (Class I, Level of Evidence: A) and plain old balloon 104 angioplasty, BMS, or DES to treat DES-ISR (Levine et al., 2011). The guideline does not issue 105 any recommendations for DEB. In real-world practice, the use of DEB for either BMS or DES restenosis showed good clinical results (Stella et al., 2011; Lee et al., 2016). DEBs provide advantages over DESs, such as rapid release of drug to the arterial wall, the absence of polymers and stent structures, and the absence of stent thrombosis (Waksman \& Pakala, 2009). Paclitaxel has been identified as the primary drug for use in DEBs because of its long-lasting antiproliferative effect and retained uptake by vascular smooth muscle cells up to 1 week

111 (Waksman \& Pakala, 2009). On the other hand, DEB also has been applied for de novo coronary

112 lesions, small vessel disease, long lesions, and bifurcations, and presented good results (Fröhlich 113 et al., 2013; Vaquerizo et al., 2015; Richelsen, Overvad \& Jensen, 2016).

114 However, there are few data about use of DEB in acute coronary syndromes, especially acute 115 myocardial infarction (AMI). One DEB-AMI trial (Belkacemi et al., 2012) in ST-segment

116 elevation myocardial infarction (STEMI) patients, angiographic results of DES were superior to 117 both BMS and DEB plus BMS. However, no clinical outcomes of DEB for STEMI and no other 118 randomized trials about DEB use in non-ST-segment elevation MI (NSTEMI) were achieved.

119 Most trials and real-world practice also use DEB for relative stable conditions such as stable 120 angina and unstable angina (Wöhrle et al., 2013; Lee et al., 2016; Fröhlich et al., 2013). 
121 Therefore, we focused on the use of DEB for acute conditions with thrombus. This study's

122 aim was to compare the differences in clinical outcomes between the use of DES and DEB for

123 AMI with ISR.

124

125 Materials and Methods

126 The study was approved by the Institutional Review Committee on Human Research of Chang

127 Gung Memorial Hospital for retrospective analysis in consecutive patients with AMI including

128 STEMI and NSTEMI who underwent PCI with DEB and DES for ISR between November 2011

129 and December 2015 in our hospital. The approval number was 201701790B0. The raw data was

130 from myocardial infarction registry of Kaohsiung Chang Gung Memorial Hospital.

131

132 Patients and groups

133 Between November 2011 and December 2015, 117 consecutive patients with AMI and received

134 PCI for ISR were retrospectively enrolled. All patients refused CABG due to high operation risk 135 and patients' choice. The patients were divided into two groups: (1) PCI with further DEB, and 136 (2) PCI with further DES. In both groups, dual anti-platelet therapy (Aspirin plus clopidogrel or 137 ticagrelor) was used for one year for AMI. The decision of using DEB or DES was on operator's 138 discretion. The most reasons that operator choose DEB were: 1) The lesions had more than two 139 layers of metallic stents. 2) The lesions were relative less plaque burden after balloon angioplasty.

\section{Endpoints}

143 Clinical outcomes such as target lesion revascularization (TLR), target vessel revascularization 144 (TVR), recurrent MI, stroke, cardiovascular mortality, and all-cause mortality were analyzed. In- 
145 hospital major adverse cardiac cerebral events (MACCEs), cardiovascular mortality, and all146 cause mortality were compared between the two groups.

147

148 Definitions

149 AMI definitions were in accordance with the most recent universal definition of AMI (Thygesen 150 et al., 2012). TLR was defined as a repeat PCI or CABG for a lesion in the previously treated 151 segment or in an adjacent $5 \mathrm{~mm}$ segment. TVR was defined as a repeat PCI in a target vessel. 152 MACCEs included TLR, TVR, recurrent MI, stroke, and cardiovascular mortality.

153 Cardiovascular mortality was defined as death related to cardiac arrhythmia, heart failure, and 154 cardiogenic shock. All-cause mortality was defined as death from any cause.

155

\section{Study endpoints}

157 The primary endpoints were a MACCE during the follow-up period. The secondary endpoint 158 was all-cause mortality during the follow-up period.

159

\section{Statistical analysis}

161 Data are expressed as a mean \pm standard deviation for continuous variables or as counts and 162 percentages for categorical variables. Continuous variables were compared using an independent sample t or Mann-Whitney U tests. Categorical variables were compared using a Chi-square statistic. A Kaplan-Meier curve was performed with log rank test for TLR, TVR, recurrent MI, and cardiovascular mortality in DEB and DES groups during the 1-year follow-up period.

Because the patients were not randomly assigned, there was some bias in baseline characteristics. In order to compare the clinical effect between DEB and DES, a propensity score matched analysis was performed as a 1-to-1 matched analysis using a logistic regression model 
169 for the DEB group versus the DES group to adjust for differences in baseline characteristics.

170 Using the estimated logits, the DEB group and the DES group had the closest estimated logit

171 value. The baseline covariates were compared between these two groups and were similar.

172 All statistical analyses were performed using SPSS 22.0 (IBM Corp., Armonk, NY). A p-

173 value $<0.05$ was considered statistically significant.

174

175 Results

176 Patient characteristics (Table 1)

177 The average age of the patients in both groups was similar, but the percentage of male was lower 178 in the DEB group (61.3\% vs. 83.3\%; $\mathrm{p}=0.021)$. Most patients presented NSTEMI in the DEB 179 and DES groups (90.7\% vs. 83.3\%), and most patients presented Killip I status. The risk factors 180 for AMI were similar between the two groups. The level of serum creatinine was similar in the 181 both groups $(2.08 \pm 1.09 \mathrm{mg} / \mathrm{dL}$ vs. $1.46 \pm 0.82 \mathrm{mg} / \mathrm{dL} ; \mathrm{p}=0.132)$. Similar prevalence of multiple 182 vessel coronary artery disease (96.0\% vs. $90.5 \%)$ and left main coronary artery disease $(30.7 \%$ 183 vs. $28.6 \%$ ) was observed in both groups. The previous stents and the infarcted artery were 184 similar between groups. The prevalence of follow-up coronary angiography was $45.3 \%$ in the 185 DEB group and $54.8 \%$ in the DES group $(\mathrm{p}=0.343)$.

186

\section{Lesion characteristics (Table 2)}

188 Most lesions were diffuse (63.1\% vs. 59.3\%) in both groups. Greater pre-PCI stenotic percentage $189(82.84 \pm 12.33 \%$ vs. $78.55 \pm 8.99 \% ; \mathrm{p}=0.025)$ and greater post-PCI stenotic percentage $(16.68 \pm$ $1908.88 \%$ vs. $12.03 \pm 6.10 \% ; p=0.001)$ were seen in the DEB group. Lower post-PCI minimal

191 luminal diameter (MLD) $(2.50 \pm 0.55 \mathrm{~mm}$ vs. $2.89 \pm 0.56 \mathrm{~mm} ; \mathrm{p}<0.001)$ and post-PCI reference 192 luminal diameter (RLD) $(3.05 \pm 0.65 \mathrm{~mm}$ vs. $3.29 \pm 0.60 \mathrm{~mm} ; \mathrm{p}=0.028)$ were seen in the DEB 
193 group. The percentage of intravascular ultrasound study (IVUS) use was similar in both groups $194(32.0 \%$ vs. $38.9 \%$; $=0.480)$. Smaller diameter of the size of DEB $(3.08 \pm 0.42 \mathrm{~mm}$ vs. $3.23 \pm$ $1950.43 \mathrm{~mm} ; \mathrm{p}=0.042)$ and similar length of the DEB $(26.41 \pm 4.20 \mathrm{~mm}$ vs. $28.02 \pm 7.81 \mathrm{~mm}$; $196 \mathrm{p}=0.095)$ were noted when comparing with the size of DES.

197

198

One-year clinical outcomes of DEB and DES group (Table 3)

199

200

201

202

203

204

205

206

207

208

209

210

211

212

213

214

215

216

Between two groups, the incidence of events including in-hospital MACCE, total MACCE, TLR, TVR, recurrent MI, stroke, cardiovascular death, and all-cause mortality were similar.

\section{The Kaplan-Meier curves of 1-year clinical outcomes of DEB and DES groups in TLR,} TVR, recurrent MI, and cardiovascular mortality (Fig 1)

In figure 1A, the Kaplan-Meier curve of 1-year TLR showed significant difference at the halfyear follow-up period (6.3\% vs. 20.9\%; $\mathrm{p}=0.034)$, and became no difference at the 1 -year follow-up period (26.9\% vs. 22.5\%; p=0.862). In figure 1B, the Kaplan-Meier curve of 1-year TVR showed no significant difference at the half-year follow-up period (11.1\% vs. $23.3 \%$; $\mathrm{p}=0.114)$, and at 1 -year follow-up period $(31.3 \%$ vs. $27.5 \%$; $=0.776)$. In figure $1 \mathrm{C}$, the KaplanMeier curve of recurrent MI showed no significant difference at the half-year follow-up period (5.1\% vs. $14.7 \% ; p=0.136)$, and at the 1 -year follow-up period $(16.1 \%$ vs. $21.9 \% ; p=0.400)$. In

figure 1D, the Kaplan-Meier curve of cardiovascular death showed no significant difference at the half-year follow-up period (6.5\% vs. $12.5 \%$; $=0.309)$, and at the 1 -year follow-up period (11.7\% vs. $12.8 \% ; p=0.765)$. 
217 After propensity score matched, all baseline characteristic became similar between two groups

218 except post-PCI MLD. In addition, higher incidence of TLR (4.9\% vs. $22.0 \%$; $=0.048)$ and

219 TVR (4.9\% vs. $24.9 \%$; $\mathrm{p}=0.026)$ was noted at the half-year follow-up period.

\section{Discussion}

222 In the present study, the baseline characteristics were similar, expect for gender. Most patients

223 had multiple vessel coronary artery disease and had undergone BMS implantation previously.

224 Most ISR were diffuse lesions in both groups. DEB group had worse pre-PCI stenotic percentage

225 and worse post-PCI results than the DES group. These results were related to no metallic

226 structure in the DEB group, but did not influence clinical outcomes. The DEB group experienced

227 less recurrent MI during the half-year and 1-year follow-up periods due to no metallic structure.

228 In the DEB group, better results at about half-year TLR and half-year TVR were noted, but

229 similar results at about 1-year TLR and TVR were noted. A relatively lower percentage of IVUS

230 use was related to an emergent condition. According to our results, use of DEB for ISR with

231 AMI had similar results as use of DES, and could decrease the possibilities of short-term events.

232 Paclitaxel is the most effective drug used with DEB technology due to its significant lipophilia,

233 which allows for a more homogeneous distribution through the vessel wall, as well as a quick

234 absorption and the duration of the effect, which may be extended for several days (Waksman \&

235 Pakala, 2009). Many studies including randomized controlled trials and meta-analyses showed

236 good results of DEB for ISR compared with conventional balloon angioplasty (Habara et al.,

237 2011; Indermuehle et al., 2013), and similar results of DEB for ISR compared with DES

238 (Alfonso et al., 2014). Most patients presented a relatively stable condition such as silent

239 ischemia, stable angina, and unstable angina in randomized controlled trials (Habara et al., 2011;

240 Indermuehle et al., 2013; Alfonso et al., 2014). However, there are few data about DEB in acute 
241 coronary syndromes, especially AMI. Compared with BMS alone for NSTEMI, patients treated 242 with BMS plus DEB had significantly less luminal loss, but the treatment did not affect patient

243 clinical outcomes (Besic et al., 2015). In STEMI, angiographic results of DES were superior to

244 both BMS and DEB plus BMS (Belkacemi et al., 2012). Therefore, physicians preferred to use

245 the metallic stent first for AMI due to high thrombus condition, and used the DEB to mimic DES.

246 Currently, no head-to-head randomized study has been performed to compare the clinical

247 outcome between only DEB and DES for AMI.

248 DES improved the outcome of AMI after PCI, but recurrent ISR and stent thrombosis still

249 were difficult problems. "Stent in stent" treatment increases the possibilities of stent thrombosis

250 due to luminal loss, chronic inflammation and hypersensitivity reactions (Alfonso et al., 2014).

251 Repeat stenting for ISR may be related to insufficient stent expansion and suboptimal stent

252 geometry because restenotic or thrombosed stents are difficult to reopen (Seedial et al., 2013).

253 DEBs have recently had a potential to overcome the limitations of DESs. Some limitations of

254 DESs are the need for long lengths to cover the entire surface of a diseased vessel, their

255 association with excessive intimal hyperplasia, and difficulties about adaptive remodeling of

256 restenosis (Seedial et al., 2013). In the present study, short-term data also showed higher

257 incidence of TLR, TVR, and recurrent MI in the DES group. Therefore, DEBs have emerged as a

258 potential alternative to the current treatment of ISR, and provide the freedom of polymers and

259 stent structures. Therefore, DEBs prevent the problem of "stent in stent", do not cause stent

260 thrombosis and luminal loss, and may decrease the possibility of sudden death if acute stent

261 thrombosis happens after repeat stenting. In addition, few have reported about DEB use for

262 combined ISR and acute coronary syndrome, especially AMI with high thrombus contained

263 condition.

264 In the present study, the patients in both groups had similar baseline characteristics, even 
265 though non-randomized controlled study. Use of DEBs seems to provide good short-term 266 outcomes and less TLR, TVR, and recurrent MI due to no stent thrombosis. However, the

267 clinical outcome became similar at 1-year follow-up period. In both groups, a relatively higher

268 event rate was noted, because the study population experienced recurrent ISR and had multiple 269 comorbidities including diabetes, and ESRD, and multiple vessel coronary artery disease. CABG

270 could be an option for the patient experienced recurrent ISR and had multiple vessel coronary

271 artery disease. In this study, all patients refused CABG due to high operation risk and patients'

272 choice. In this study, we focused on the impact of DEB for the combination of AMI and ISR in

273 clinical practice for high risk population.

274

275 Limitations

276 The present study had some limitations, including being a non-randomized study and having 277 selection bias because the operator may consider the use of DES in the patients with complex 278 lesions and the use of DEB in the patients with shorter and simple lesions. Even though the 279 present study is non-randomized, the baseline characteristics were very similar between the two 280 groups. In addition, no previous study compared clinical outcomes between only DEB and DES 281 use for AMI with ISR. Our study provided the insight on the use of DEB for AMI in clinical 282 practice for high risk population.

283

284 Conclusions

285 DEB is a reasonable strategy for AMI with ISR. Compared with DES, DEB was an alternative 286 strategy which yielded acceptable short-term outcomes and similar 1-year clinical outcomes. 
References

Wöhrle J, Zadura M, Möbius-Winkler S, Leschke M, Opitz C, Ahmed W, Barragan

P, Simon JP, Cassel G, Scheller B. 2012. SeQuentPlease World Wide Registry: clinical results of SeQuentPlease paclitaxel-coated balloon angioplasty in large-scale, prospective registry study.

310 Journal of the American College of Cardiology 60:1733-1738.

311 Authors/Task Force members, Windecker S, Kolh P, Alfonso F, Collet JP, Cremer J, Falk

312 V, Filippatos G, Hamm C, Head SJ, Jüni P, Kappetein AP, Kastrati A, Knuuti J, 
313 Landmesser U, Laufer G, Neumann FJ, Richter DJ, Schauerte P, Sousa Uva M, Stefanini

314 GG, Taggart DP, Torracca L, Valgimigli M, Wijns W, Witkowski A. 2014. 2014

315 ESC/EACTS Guidelines on myocardial revascularization: The Task Force on Myocardial

316 Revascularization of the European Society of Cardiology (ESC) and the European Association

317 for Cardio-Thoracic Surgery (EACTS)Developed with the special contribution of the European

318 Association of Percutaneous Cardiovascular Interventions (EAPCI). European Heart Journal 35:

$319 \quad 2541-2619$.

320 Levine GN, Bates ER, Blankenship JC, Bailey SR, Bittl JA, Cercek B, Chambers CE, Ellis

321 SG, Guyton RA, Hollenberg SM, Khot UN, Lange RA, Mauri L, Mehran R, Moussa ID,

322 Mukherjee D, Nallamothu BK, Ting HH. 2011. 2011 ACCF/AHA/SCAI Guideline for

323 Percutaneous Coronary Intervention: executive summary: a report of the American College of

324 Cardiology Foundation/American Heart Association Task Force on Practice Guidelines and the

325 Society for Cardiovascular Angiography and Interventions. Circulation 124:2574-2609.

326 Stella PR, Belkacemi A, Waksman R, Stahnke S, Torguson R, von Strandmann RP,

327 Agostoni P, Sangiorgi G, Silber S; Valentine Investigators. 2011. The Valentines Trial:

328 results of the first one week worldwide multicentre enrolment trial, evaluating the real world

329 usage of the second generation DIOR paclitaxel drug eluting balloon for in-stent restenosis

330 treatment. EuroIntervention 7:705-710.

331 Lee WC, Fang YN, Fang CY, Chen CJ, Yang CH, Yip HK, Hang CL, Wu CJ, Fang HY.

332 2016. Comparison of Clinical Results Following the Use of Drug-Eluting Balloons for a Bare-

333 Metal Stent and Drug-Eluting Stent Instent Restenosis. Journal of Interventional Cardiology

334 29:469-479.

335 Waksman R, Pakala R. 2009. Balloon drug-eluting: the comeback kid? Circulation:

336 Cardiovascular Interventions 2:352-358. 
337 Fröhlich GM, Lansky AJ, Ko DT, Archangelidi O, De Palma R, Timmis A, Meier P. 2013.

338 Drug eluting balloons for de novo coronary lesions - a systematic review and meta-analysis.

339 BMC Medicine 11:123.

340 Vaquerizo B, Miranda-Guardiola F, Fernández E, Rumoroso JR, Gómez-Hospital JA,

341 Bossa F, Iñiguez A, Oategui I, Serra A. 2015. Treatment of Small Vessel Disease With the

342 Paclitaxel Drug-Eluting Balloon: 6-Month Angiographic and 1-Year Clinical Outcomes of the

343 Spanish Multicenter Registry. Journal of Interventional Cardiology 28:430-438.

344 Richelsen RK, Overvad TF2,3, Jensen SE. 2016. Drug-Eluting Balloons in the Treatment of

345 Coronary De Novo Lesions: A Comprehensive Review. Cardiology and Therapy 5:133-160.

346 Belkacemi A, Agostoni P, Nathoe HM, Voskuil M, Shao C, Van Belle E, Wildbergh T,

347 Politi L, Doevendans PA, Sangiorgi GM, Stella PR. 2012. First results of the DEBAMI (drug

348 eluting balloon in acute ST-segment elevation myocardial infarction) trial: a multicentre

349 randomized comparison of drug-eluting balloon plus bare-metal stent versus bare-metal stent

350 versus drug-eluting stent in primary percutaneous coronary intervention with 6-month

351 angiographic, intravascular, functional, and clinical outcomes. Journal of the American College

352 of Cardiology 59;2327-2337.

353 Thygesen K, Alpert JS, Jaffe AS, Simoons ML, Chaitman BR, White HD; Joint

354 ESC/ACCF/AHA/WHF Task Force for the Universal Definition of Myocardial

355 Infarction, Katus HA, Lindahl B, Morrow DA, Clemmensen PM, Johanson P, Hod

356 H, Underwood R, Bax JJ, Bonow RO, Pinto F, Gibbons RJ, Fox KA, Atar D, Newby

357 LK, Galvani M, Hamm CW, Uretsky BF, Steg PG, Wijns W, Bassand JP, Menasché

358 P, Ravkilde J, Ohman EM, Antman EM, Wallentin LC, Armstrong PW, Simoons

359 ML, Januzzi JL, Nieminen MS, Gheorghiade M, Filippatos G, Luepker RV, Fortmann

360 SP, Rosamond WD, Levy D, Wood D, Smith SC, Hu D, Lopez-Sendon JL, Robertson 
361 RM, Weaver D, Tendera M, Bove AA, Parkhomenko AN, Vasilieva EJ, Mendis S. 2012.

362 Third universal definition of myocardial infarction. Circulation 126:2020-2035.

363 Habara S, Mitsudo K, Kadota K, Goto T, Fujii S, Yamamoto H, Katoh H, Oka N, Fuku Y,

364 Hosogi S, Hirono A, Maruo T, Tanaka H, Shigemoto Y, Hasegawa D, Tasaka H, Kusunose

365 M, Otsuru S, Okamoto Y, Saito N, Tsujimoto Y, Eguchi H, Miyake K, Yoshino M. 2011.

366 Effectiveness of paclitaxel-eluting balloon catheter in patients with sirolimus-eluting stent

367 restenosis. Journal of the American College of Cardiology Cardiovascular Intervention 4:149368154.

369 Indermuehle A, Bahl R, Lansky AJ, Froehlich GM, Knapp G, Timmis A, Meier P. 2013.

370 Drug-eluting balloon angioplasty for in-stent restenosis: a systematic review and meta-analysis

371 of randomized controlled trials. Heart 99:327-333.

372 Alfonso F, Pérez-Vizcayno MJ, Cárdenas A, García Del Blanco B, Seidelberger B, Iñiguez

373 A, Gómez-Recio M, Masotti M, Velázquez MT, Sanchís J, García-Touchard A, Zueco J,

374 Bethencourt A, Melgares R, Cequier A, Dominguez A, Mainar V, López-Mínguez JR,

375 Moreu J, Martí V, Moreno R, Jiménez-Quevedo P, Gonzalo N, Fernández C, Macaya C;

376 RIBS V Study Investigators, under the auspices of the Working Group on

377 Interventional Cardiology of the Spanish Society of Cardiology. 2014. A randomized

378 comparison of drug-eluting balloon versus everolimus-eluting stent in patients with bare-metal

379 stent-in-stent restenosis: the RIBS V Clinical Trial (Restenosis Intra-stent of Bare Metal Stents:

380 paclitaxel-eluting balloon vs. everolimus-eluting stent). Journal of the American College of

381 Cardiology 63:1378-1386.

382 Besic KM, Strozzi M, Margetic E, Bulum J, Kolaric B. 2015. Drug eluting balloons in

383 patients with non-ST elevation acute coronary syndrome. Journal of Cardiology 65:203-207.

384 Alfonso F, Byrne RA, Rivero F, Kastrati A. Current treatment of in-stent restenosis. Journal of 
385 the American College of Cardiology 63:2659-2673.

386 Seedial SM${ }^{1}$, Ghosh S, Saunders RS, Suwanabol PA, Shi X, Liu B, Kent KC. 2013. Local

387 drug delivery to prevent restenosis. Journal of Vascular Surgery 57:1403-1414.

388

389

390

391

392

393

394

395 
396 Fig 1. The Kaplan-Meier curves of 1-year clinical outcomes of DEB and DES group in TLR, 397 TVR, recurrent MI, and cardiovascular mortality

398 Figure 1A: The Kaplan-Meier curve of 1-year TLR: Significant difference at the half-year 399 follow-up period ( $6.3 \%$ vs. $20.9 \%$; p=0.034) was noted. No difference at 1 -year follow-up period $400 \quad(26.9 \%$ vs. $22.5 \% ; p=0.862)$ was noted.

401

402 Figure 1B: The Kaplan-Meier curve of 1-year TVR: No significant difference was noted at the 403 half-year follow-up period (11.1\% vs. $23.3 \%$; $=0.114)$, and at the 1-year follow-up period $404 \quad(31.3 \%$ vs. $27.5 \% ; p=0.776)$.

405

406 Figure 1C: The Kaplan-Meier curve of 1-year recurrent myocardial infarction: No significant 407 difference was noted at at the half-year follow-up period (5.1\% vs. $14.7 \%$; $=0.136)$, and at 1 408 year follow-up period (16.1\% vs. $21.9 \%$; $=0.400)$.

409

410 Figure 1D: The Kaplan-Meier curve of 1-year cardiovascular mortality: The Kaplan-Meier curve 411 of 1-year TVR: No significant difference was noted at the half-year follow-up period (6.5\% vs. $41212.5 \% ; p=0.309)$, and at 1 -year follow-up period $(11.7 \%$ vs. $12.8 \% ; p=0.765)$. 
Table $\mathbf{1}$ (on next page)

Patient characteristics of DEB and DES group 
1 Table 1

2 Patient characteristics of DEB and DES group

DEB DES P value

$(\mathrm{N}=75 ; \mathrm{L}=103)(\mathrm{N}=42 ; \mathrm{L}=54)$

\section{General demographics}

Age (years)

Male sex $(\%)$

Indication

STEMI (\%)

NSTEMI (\%)

Killip classification

I $(\%)$

II $(\%)$

III $(\%)$

IV $(\%)$

\section{Risk factors for MI}

Diabetes (\%)

Current smoker (\%)

Hypertension (\%)

Prior MI (\%)

Prior stroke (\%)

PAOD (\%)

Dyslipidemia (\%)

CABG (\%)

ESRD on maintenance hemodialysis (\%) 28 (37.3)

Heart failure (\%)

46 (61.3)

7 (9.3)

68 (90.7)

34 (45.3)

13 (17.3)

17 (22.7)

$11(14.7)$

58 (77.3)

25 (33.3)

64 (85.3)

32 (42.7)

8 (10.7)

$16(21.3)$

39 (52.0)

$5(6.7)$

34 (45.3)
$67.53 \pm 11.62$

\section{Laboratory examination}

Creatinine (except ESRD) (mg/dL)

CK-MB (ng/mL)

Troponin-I (ng/mL)

$\begin{array}{lll}2.08 \pm 1.09 & 1.46 \pm 0.82 & 0.132 \\ 51.57 \pm 11.84 & 76.59 \pm 15.95 & 0.166 \\ 18.07 \pm 8.59 & 24.72 \pm 10.86 & 0.244\end{array}$

$69.86 \pm 11.00$

35 (83.3)

$6(14.3)$

$36(85.7)$

0.292

0.021

0.541

0.079

$29(69.0)$

$4(9.5)$

7 (16.7)

$2(4.8)$

$26(61.9) \quad 0.089$

$16(38.1) \quad 0.687$

$37(88.1) \quad 0.784$

$15(35.7) \quad 0.556$

$7(16.7) \quad 0.394$

$4(9.5) \quad 0.129$

$23(54.8) \quad 0.848$

$3(7.1) \quad 1.000$

$12(28.6) \quad 0.418$

$19(45.2) \quad 1.000$ 
Left ventricular ejection fraction (\%)

Before

After

$$
\begin{array}{ll}
55.47 \pm 12.06 & 51.42 \pm 12.79 \\
59.26 \pm 11.75 & 55.13 \pm 13.08
\end{array}
$$

\section{Characteristics of coronary artery disease}

Single or multiple-vessel disease

Single vessel disease (\%)

$3(4.0)$

$4(9.5)$

Double vessel disease (\%)

$16(21.3)$

Triple vessel disease (\%)

$56(74.7)$

33 (78.6)

Left main disease (\%)

$23(30.7)$

$12(28.6)$

0.837

Previous stent

Bare-metal stent (\%)

59 (57.3)

30 (55.6)

Drug-eluting stent (\%)

$44(42.7)$

24 (44.4)

\section{Infarcted artery}

Left main (\%)

$1(1.0)$

$3(5.6)$

Left anterior descending artery $(\%)$

$46(44.7)$

$21(38.9)$

Left circumflex artery (\%)

$25(24.3)$

$9(16.7)$

Right coronary artery (\%)

$31(30.1)$

$21(38.9)$
0.867

0.174
0.343

$\begin{array}{llll}\text { One-year follow-up angiography (\%) } & 34(45.3) & 23(54.8) & 0.343\end{array}$

3 Data are expressed as mean \pm SD or as number (percentage).

4 *Abbreviation: N: number; L: lesion; DEB: drug-eluting balloon; DES: drug-eluting stent;

5 STEMI: ST-segment elevation myocardial infarction; NSTEMI: non ST-segment elevation

6 myocardial infarction; MI: myocardial infarction; PAOD: peripheral arterial occlusive disease;

7 CABG: coronary artery bypass grafting; ESRD: end stage renal disease; CK-MB: creatine 8 kinase-MB. 
Table 2 (on next page)

Lesion characteristics of DEB and DES group 
1 Table 2

2 Lesion characteristics of DEB and DES group

\begin{tabular}{lll} 
DEB & DES & P value \\
$(\mathrm{N}=75 ; \mathrm{L}=103)$ & $(\mathrm{N}=42 ; \mathrm{L}=54)$ & \\
\hline
\end{tabular}

Lesion type

Focal lesion (\%)

38 (36.9)

0.730

Diffuse lesion (\%)

$65(63.1)$

$22(40.7)$

$32(59.3)$

The characteristics of lesion

Pre-PCI stenosis (\%)

$82.84 \pm 12.33 \quad 78.55 \pm 8.99 \quad 0.025$

$\operatorname{MLD}(\mathrm{mm})$

$\begin{array}{lll}0.52 \pm 0.40 & 0.60 \pm 0.30 & 0.185\end{array}$

$\operatorname{RLD}(\mathrm{mm})$

$2.93 \pm 0.65$

$2.88 \pm 0.63$

0.668

Post-PCI stenosis (\%)

$16.68 \pm 8.88$

$12.03 \pm 6.10$

0.001

$\operatorname{MLD}(\mathrm{mm})$

$2.50 \pm 0.55$

$2.89 \pm 0.56$

$<0.001$

RLD (mm)

$3.05 \pm 0.65$

$3.29 \pm 0.60$

0.028

The use of intravascular ultrasound study 33 (32.0)

$21(38.9)$

0.480

(\%)

\section{The characteristics of DEB or DES}

Diameter (mm)

$\begin{array}{lll}3.08 \pm 0.42 & 3.23 \pm 0.43 & 0.042 \\ 26.41 \pm 4.20 & 28.02 \pm 7.81 & 0.095\end{array}$

Length (mm)

$26.41 \pm 4.20$

$28.02 \pm 7.81$

0.095

3 Data are expressed as mean $\pm \mathrm{SD}$ or as number (percentage).

4 *Abbreviation: N: number; L: lesion; DEB: drug-eluting balloon; DES: drug-eluting stent; PCI:

5 percutaneous coronary intervention; MLD: minimal luminal diameter; RLD: reference luminal 6 diameter. 
Table 3(on next page)

One-year clinical outcomes of DEB and DES group 
1 Table 3

2 One-year clinical outcomes of DEB and DES group

\begin{tabular}{llll}
\hline & DEB & DES & P value \\
& $(\mathrm{N}=75 ; \mathrm{L}=103)$ & $(\mathrm{N}=42 ; \mathrm{L}=54)$ & \\
\hline In-hospital MACCE (\%) & $2(2.7)$ & $3(7.1)$ & 0.348 \\
MACCE (\%) & $24(34.0)$ & $15(35.7)$ & 0.688 \\
Target-lesion revascularization (\%) & $21(26.9)$ & $9(22.5)$ & 0.661 \\
Target-vessel revascularization (\%) & $25(31.3)$ & $11(27.5)$ & 0.833 \\
Recurrent myocardial infarction (\%) & $9(16.1)$ & $7(21.9)$ & 0.570 \\
$\quad$ STEMI (\%) & 0 & $1(14.3)$ & \\
$\quad$ NSTEMI (\%) & $9(100)$ & $6(85.7)$ & \\
Stroke (\%) & $2(3.6)$ & $1(3.1)$ & 1.000 \\
Cardiovascular mortality (\%) & $7(11.7)$ & $5(12.8)$ & 1.000 \\
All-cause mortality (\%) & $16(22.9)$ & $7(17.1)$ & 0.628 \\
\hline
\end{tabular}

3 Data are expressed as mean $\pm \mathrm{SD}$ or as number (percentage).

4 *Abbreviation: N: number; L: lesion; DEB: drug-eluting balloon; DES: drug-eluting stent;

5 MACCE: major adverse cardiac cerebral event; STEMI: ST-segment elevation myocardial

6 infarction; NSTEMI: non ST-segment elevation myocardial infarction. 


\section{Table 4(on next page)}

Patient and lesion characteristics of DEB and DES group after propensity score matched 
$1 \quad$ Table 4

2 Patient and lesion characteristics of DEB and DES group after propensity score matched

DEB DES P value

$(\mathrm{N}=40 ; \mathrm{L}=52) \quad(\mathrm{N}=40 ; \mathrm{L}=52)$

General demographics

Age (years)

Male sex $(\%)$

Indication

STEMI $(\%)$

NSTEMI (\%)

Killip classification

$\geqq$ III $(\%)$

Risk factors for MI

Diabetes (\%)

Current smoker $(\%)$

Hypertension (\%)

Prior MI (\%)

Prior stroke (\%)

PAOD (\%)

Dyslipidemia (\%)

CABG (\%)

ESRD on maintenance hemodialysis (\%)

Heart failure (\%)

Laboratory examination

CK-MB (ng/mL)

Troponin-I (ng/mL)

Left ventricular ejection fraction (\%)

Before

After

Characteristics of coronary artery disease
$10(25.0)$

$9(22.5)$

1.000

$67.78 \pm 13.04 \quad 69.48 \pm 11.00 \quad 0.530$

$34(85.0) \quad 33(82.5) \quad 1.000$

$3(7.5) \quad 6(15.0)$

$37(92.5) \quad 34(85.0)$

0.481

$\begin{array}{lll}24(60.0) & 26(65.0) & 0.818 \\ 20(50.0) & 15(37.5) & 0.367 \\ 35(87.5) & 35(87.5) & 1.000 \\ 15(37.5) & 13(32.5) & 0.815 \\ 5(12.5) & 7(17.5) & 0.755 \\ 6(15.0) & 4(10.0) & 0.737 \\ 24(60.0) & 23(57.5) & 1.000 \\ 4(10.0) & 3(7.5) & 1.000 \\ 13(32.5) & 12(30.0) & 1.000 \\ 14(30.0) & 18(45.0) & 0.494\end{array}$

$58.42 \pm 17.24 \quad 68.86 \pm 19.71 \quad 0.638$

$17.92 \pm 8.91 \quad 22.45 \pm 9.75 \quad 0.492$

$53.59 \pm 11.96 \quad 50.95 \pm 13.93 \quad 0.059$

$58.94 \pm 11.84 \quad 55.72 \pm 13.19 \quad 0.137$ 
Multiple-vessel disease

Left main disease (\%)

Previous stent

Bare-metal stent (\%)

Drug-eluting stent (\%)

Infarcted artery

Left main (\%)

Left anterior descending artery (\%)

Left circumflex artery (\%)

Right coronary artery (\%)

Lesion type

Focal lesion (\%)

Diffuse lesion (\%)

The characteristics of lesion

Pre-PCI stenosis (\%)

$\operatorname{MLD}(\mathrm{mm})$

RLD (mm)

Post-PCI stenosis (\%)

$\operatorname{MLD}(\mathrm{mm})$

RLD (mm)
$30(75.0)$

$13(32.5)$

$32(80.0)$

$12(30.0)$

0.620

1.000

1.000

$30(57.7)$

$30(57.7)$

$22(42.3)$

$22(42.3)$

$0(0)$

$20(38.5)$

$12(23.1)$

$20(38.5)$

$24(46.2)$

$28(53.8)$
0.692

0.330

$3(5.8)$

$20(38.5)$

9 (17.3)

$20(38.5)$

$21(40.4)$

$31(59.6)$

$\begin{array}{llll}\text { The use of intravascular ultrasound study } 13(32.5) & 15(37.5) & 0.815\end{array}$ (\%)

\section{The characteristics of DEB or DES}

Diameter (mm)

$$
\begin{array}{lll}
3.16 \pm 0.43 & 3.23 \pm 0.44 & 0.462 \\
26.50 \pm 4.21 & 27.64 \pm 7.70 & 0.353
\end{array}
$$

Length $(\mathrm{mm})$

3 Data are expressed as mean $\pm \mathrm{SD}$ or as number (percentage).

4 *Abbreviation: N: number; L: lesion; DEB: drug-eluting balloon; DES: drug-eluting stent;

5 STEMI: ST-segment elevation myocardial infarction; NSTEMI: non ST-segment elevation

6 myocardial infarction; MI: myocardial infarction; PAOD: peripheral arterial occlusive disease;

7 CABG: coronary artery bypass grafting; ESRD: end stage renal disease; CK-MB: creatine 
8 kinase-MB; PCI: percutaneous coronary intervention; MLD: minimal luminal diameter; RLD:

9 reference luminal diameter. 


\section{Table 5 (on next page)}

One-year clinical outcomes of DEB and DES group after propensity score matched 
1 Table 5

2 One-year clinical outcomes of DEB and DES group after propensity score matched

\begin{tabular}{llll}
\hline & DEB & DES & P value \\
& $(\mathrm{N}=40 ; \mathrm{L}=52)$ & $(\mathrm{N}=40 ; \mathrm{L}=52)$ & \\
\hline $\begin{array}{l}\text { In-hospital MACCE (\%) } \\
\text { Half-year }\end{array}$ & $0(0)$ & $3(7.5)$ & 0.241 \\
Target-lesion revascularization (\%) & $2(4.9)$ & $9(22.0)$ & 0.048 \\
Target-vessel revascularization (\%) & $2(4.9)$ & $10(24.4)$ & 0.026 \\
One-year & & & \\
MACCE (\%) & $11(27.5)$ & $15(37.5)$ & 0.474 \\
Target-lesion revascularization (\%) & $14(34.1)$ & $9(23.7)$ & 0.333 \\
Target-vessel revascularization (\%) & $14(34.1)$ & $11(28.9)$ & 0.638 \\
Recurrent myocardial infarction (\%) & $3(10.0)$ & $7(23.3)$ & 0.299 \\
Stroke (\%) & $1(3.2)$ & $1(3.3)$ & 1.000 \\
Cardiovascular mortality (\%) & $0(0)$ & $5(13.5)$ & 0.060 \\
All-cause mortality (\%) & $6(16.7)$ & $7(17.9)$ & 1.000 \\
\hline
\end{tabular}

3 Data are expressed as mean $\pm \mathrm{SD}$ or as number (percentage).

4 *Abbreviation: N: number; L: lesion; DEB: drug-eluting balloon; DES: drug-eluting stent;

5 MACCE: major adverse cardiac cerebral event.

6 


\section{Figure 1 (on next page)}

The Kaplan-Meier curves of 1-year clinical outcomes of DEB and DES group in TLR, TVR, recurrent $\mathrm{MI}$, and cardiovascular mortality

A: The Kaplan-Meier curve of 1-year TLR: Significant difference at the half-year follow-up period (6.3\% vs. $20.9 \% ; p=0.034$ ) was noted. No difference at 1 -year follow-up period $(26.9 \%$ vs. $22.5 \% ; p=0.862)$ was noted.

B: The Kaplan-Meier curve of 1-year TVR: No significant difference was noted at the half-year follow-up period (11.1\% vs. $23.3 \% ; p=0.114)$, and at the 1 -year follow-up period $(31.3 \%$ vs. $27.5 \% ; p=0.776)$.

C: The Kaplan-Meier curve of 1-year recurrent myocardial infarction: No significant difference was noted at at the half-year follow-up period (5.1\% vs. $14.7 \% ; p=0.136)$, and at 1-year follow-up period (16.1\% vs. $21.9 \% ; p=0.400)$.

D: The Kaplan-Meier curve of 1-year cardiovascular mortality: The Kaplan-Meier curve of 1-year TVR: No significant difference was noted at the half-year follow-up period $(6.5 \%$ vs. $12.5 \% ; p=0.309)$, and at 1 -year follow-up period (11.7\% vs. $12.8 \% ; p=0.765)$. 


\section{PeerJ}

A

Target lesion revascularization

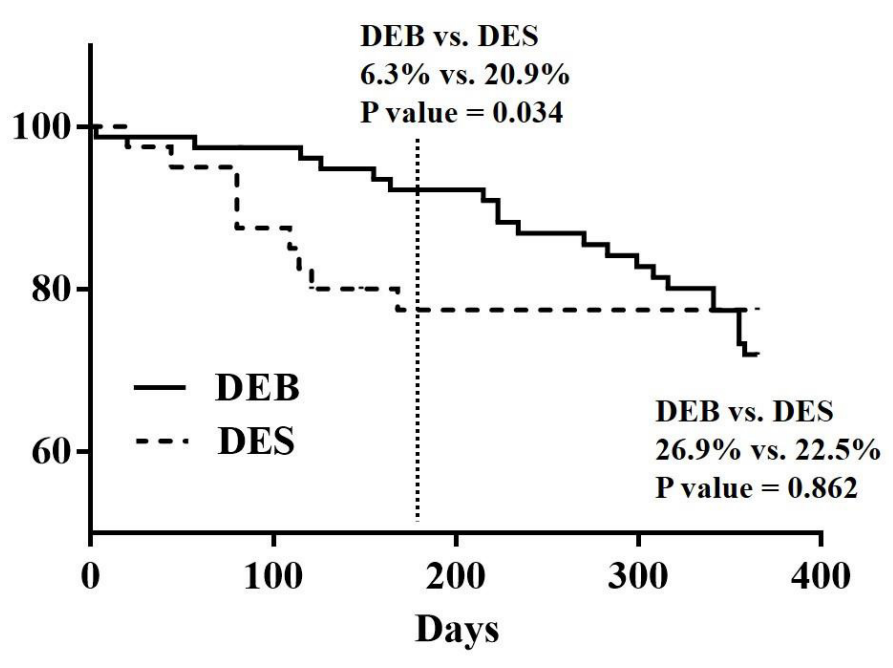

DEB

DES

C

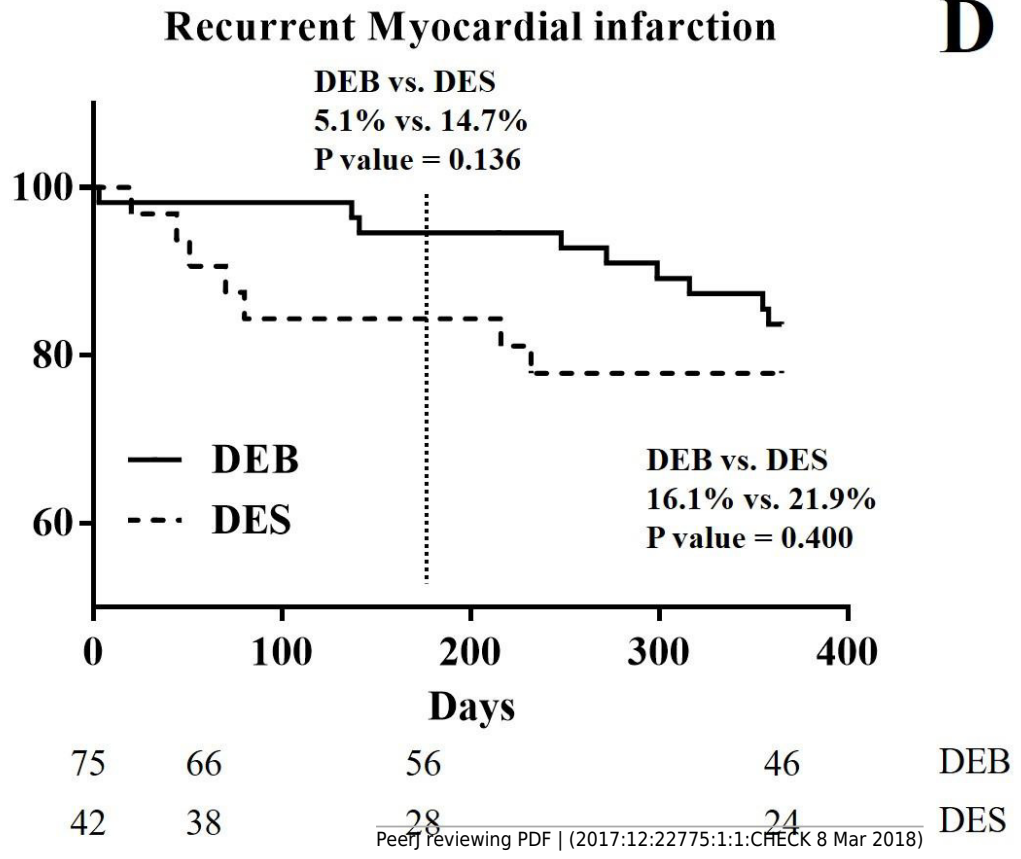

B

Manuscript to be reviewed

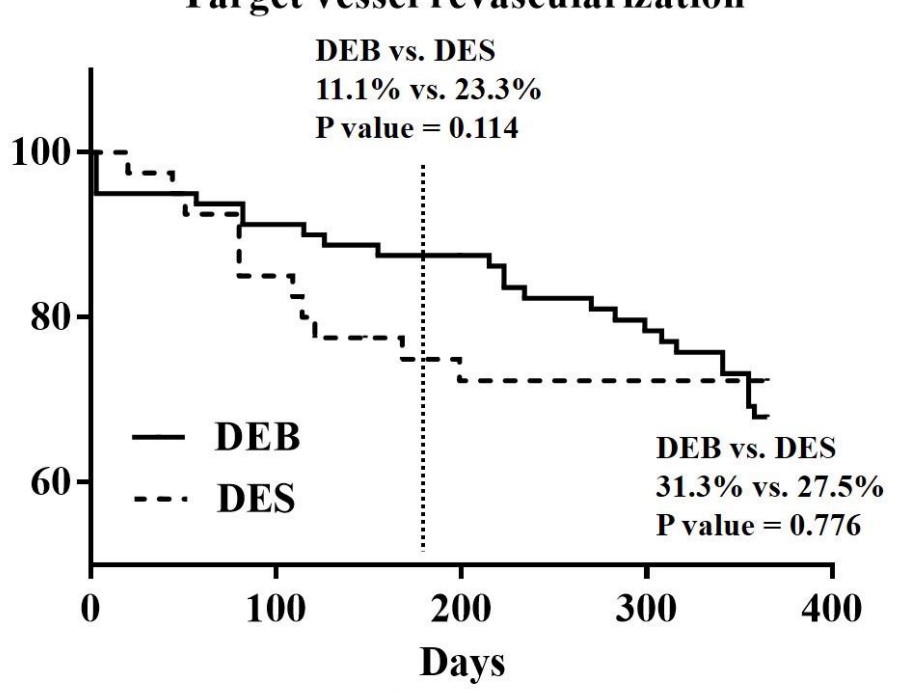

DEB

DES

$103 \quad 87$
5

72

52

$54 \quad 49$

32

28

D

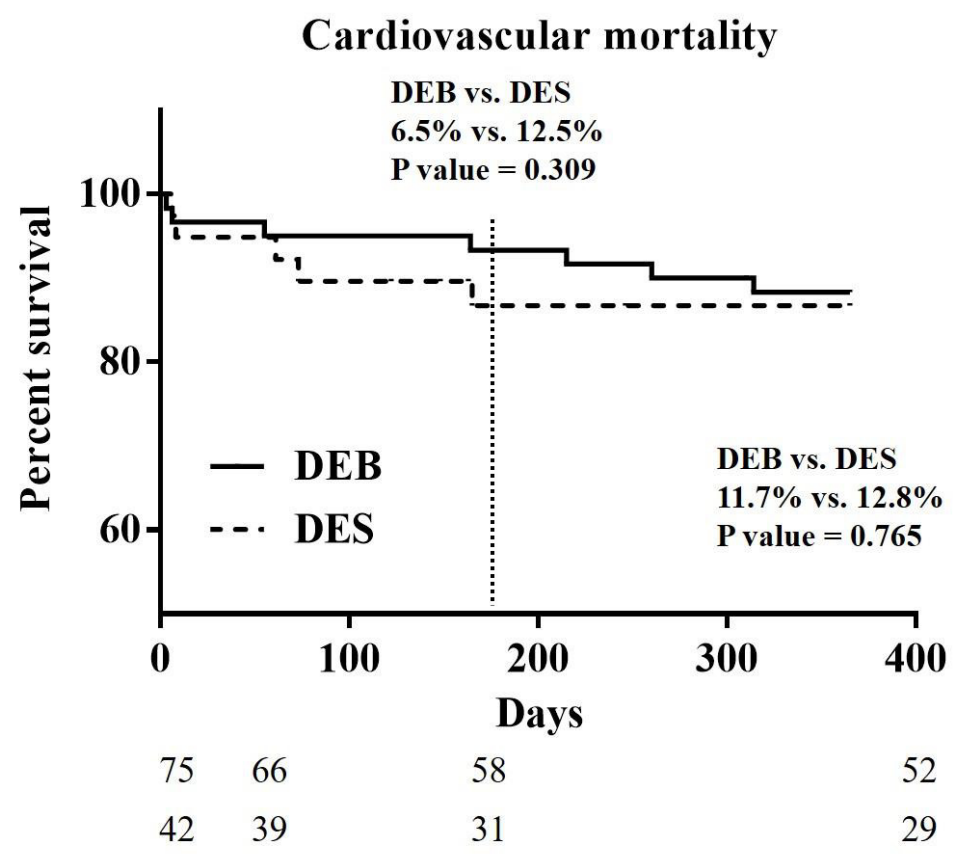

